\title{
Steps toward a universal flu vaccine
}

Influenza $\mathrm{A}$ is a highly mutable virus that infects millions of people every year and can be fatal in severe cases. Human antibodies are effective against a few strains belonging to 2 of the 16 subtypes of influenza $\mathrm{A}$, and new vaccines based on those antibodies are developed each year to match the evolved viruses, at considerable expense. Although various groups have isolated antibodies that are effective against multiple subtypes of influenza A, a vaccine that protects against all subtypes has long been sought. Development of such a vaccine requires the isolation of neutralizing antibodies that are broadly effective against these subtypes.

Now, a collaborative effort led by Antonio Lanzavecchia (Institute for Research in Biomedicine, Bellinzona, Switzerland) and John J. Skehel (Medical Research Council, London, UK) has identified an antibody that neutralizes all 16 subtypes of influenza A. The antibody has broad efficacy and high potency and therefore may be useful in developing a 'universal' vaccine.
Although it could be years before such a vaccine is designed and tested, the researchers believe that in the meantime, the antibody itself could be used as a treatment. By binding to the virus, the antibody prevents it from infecting mammalian cells. The antibody, called FI6, prevented mortality in mice and ferrets that were infected with flu. "The animals survived infection with multiple different flu strains at doses that would usually kill them," Lanzavecchia said in an interview with Nature News. "In humans, even reducing the viral load by $10 \%$ could help stop people getting sick."

The group used a new high-throughput method to screen for rare antibodies from 104,000 cultured plasma cells from eight human donors who had been infected with or immunized against multiple strains of influenza A (Science doi:10.1126/ science.1205669; published online 28 July 2011). After isolating FI6, the scientists carried out X-ray crystallography studies to analyze how the antibody bound to the

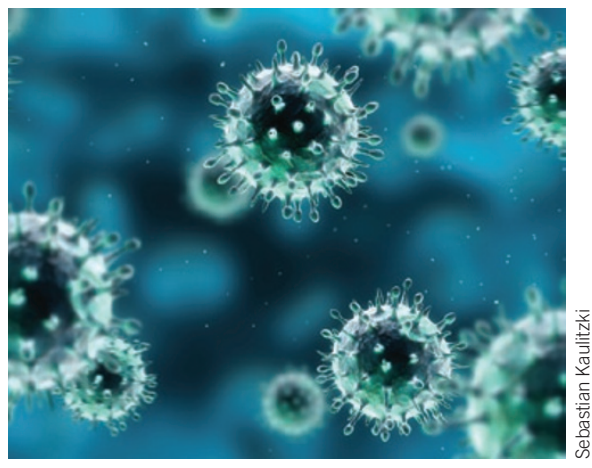

virus. They found that FI6 binds to the stem region of influenza's hemagglutinin protein, a relatively stable portion of the viral structure. In contrast, most influenza antibodies bind to the head region of hemagglutinin, which mutates frequently.

In a press release, Lanzavecchia concluded, "As the first and only antibody which targets all known subtypes of the influenza A virus, FI6 represents an important new treatment option."

Monica Harrington

\section{NEMATODES WIN AN ‘EVOLUTIONARY ARMS RACE' WITH FUNGI}

The nematode Caenorhabditis elegans is a useful model for studying a behavior or set of behaviors, because its entire sensorimotor circuit has been defined and can easily be manipulated genetically. New research takes advantage of this fact, using nematodes to study the mechanisms underlying the evolution of escape behaviors.

In the natural nematode environment, certain types of fungi prey on nematodes as their main food source and have developed distinctive trapping devices to catch them. One device utilizes a constricting ring mechanism: when a nematode passes through the ring, the friction induces the cells of the ring to rapidly inflate inwards and trap it. Unless the nematode can quickly escape from the constricting ring, it will be captured and digested by the fungi.

Mark J. Alkema and colleagues at the University of Massachusetts Medical School in Worcester, MA, examined the motor pattern required for C. elegans to escape from the fungi once caught (Curr. Biol. doi:10.1016/j.cub.2011.06.063; published online 28 July 2011). Wild-type worms that were able to escape used a behavior commonly seen in response to light touch on the nose or the front half of the body: the animal quickly moves backwards while suppressing its usual exploratory side-to-side head movements.

In order to investigate the individual components of this escape behavior, the researchers analyzed the trap encounters of several mutants with impairments in one or multiple aspects of the touch response. The ability to escape from the traps was markedly reduced in mutants with deficiencies of the gene mec-4 compared with wild-type animals. Other mutants with deficits in backwards locomotion also escaped less frequently. A mutation inactivating the gene lgc-55, which caused failure to suppress head movements, also decreased the animals' likelihood of escape. The results of these experiments show that successful escape requires a touchinduced coordination of backward movement with the suppression of side-to-side head movements.

The researchers also carried out competition experiments between wild-type and lgc-55 mutants to determine whether the suppression of head movements provides a selective advantage. They found that the mutants were more frequently captured by the ring mechanism. This raises the possibility that the touch-induced suppression of head movements may be an evolved means of preventing the activation of the ring cells, thereby increasing the likelihood of escape. This research not only provides an evolutionary explanation for the complex escape behavior of $C$. elegans but also highlights their usefulness as a research tool. 